\title{
Local consolidative therapy for oligometastatic non-small cell lung cancer
}

\author{
Pamela P. Samson ${ }^{1,2}$, Matthew B. Spraker ${ }^{1,2}$, Shahed N. Badiyan ${ }^{1,2}$, Gregory Vlacich ${ }^{1,2}$, Clifford G. \\ Robinson $^{1,2}$, Aadel A. Chaudhuri, ${ }^{1,2}$ \\ ${ }^{1}$ Department of Radiation Oncology, Washington University School of Medicine, St. Louis, MO, USA; ${ }^{2}$ Alvin J. Siteman Cancer Center, Barnes- \\ Jewish Hospital and Washington University School of Medicine, St. Louis, MO, USA \\ Correspondence to: Pamela P. Samson, MD, MPHS; Aadel A. Chaudhuri, MD, PhD. Department of Radiation Oncology, Washington University \\ School of Medicine, 660 South Euclid Ave., Campus Box 8224, St. Louis, MO 63110, USA. Email: psamson@wustl.edu; aadel@wustl.edu. \\ Provenance: This is an invited article commissioned by the Section Editor Dr. Lei Deng (Department of Medicine, Jacobi Medical Center, Albert \\ Einstein College of Medicine, Bronx, NY, USA). \\ Comment on: Gomez DR, Tang C, Zhang J, et al. Local Consolidative Therapy Vs. Maintenance Therapy or Observation for Patients With \\ Oligometastatic Non-Small-Cell Lung Cancer: Long-Term Results of a Multi-Institutional, Phase II, Randomized Study. J Clin Oncol \\ 2019;37:1558-65.
}

Submitted Oct 22, 2019. Accepted for publication Oct 31, 2019.

doi: $10.21037 /$ jtd.2019.11.19

View this article at: http://dx.doi.org/10.21037/jtd.2019.11.19

The concept of local consolidative therapy (LCT) for oligometastatic non-small cell lung cancer (NSCLC) using radiation therapy and/or surgical resection has been present for approximately two decades (1-3). However, data supporting this approach has evolved from single-institution retrospective series (which can be influenced by selection bias) to multi-institutional randomized studies. This year, Gomez and colleagues published longer term follow-up of such a trial comparing LCT versus maintenance therapy/ observation (MTO) for patients with radiologically apparent, oligometastatic NSCLC following first-line systemic therapy (4). In that analysis, it was reported that not only was the progression-free survival (PFS) significantly higher for patients receiving LCT versus MTO (median 14.2 versus 4.4 months, respectively), but LCT patients also experienced significantly longer median overall survival (OS) (median 41.2 vs. 17.0 months). There were no grade 3 or greater toxicities. These differences were detected despite the fact that almost half of the MTO patients had disease progression and received salvage LCT. However, unlike upfront LCT, salvage LCT did not lengthen median OS compared to MTO (median 17.0 months).

These findings are certainly promising and exciting in the context of other recent Phase II randomized trials. In the SABR-COMET trial, Palma and colleagues used a slightly different approach-eligible patients could have up to 5 metastatic lesions receiving definitive-intent dosing. In that Phase II study, median OS in the SBRT arm was significantly longer than in the patients receiving standardof-care palliative therapy, 41 versus 26 months (5). The idea that consolidative radiation therapy could be used to keep patients off systemic therapy following first-line treatment is attractive from many perspectives-the patient, the payer, and the physician. The differences in survival in both of these randomized trials are staggering. One wonders what the international multidisciplinary response would be if the agent being tested was a new immunologic or targeted agent rather than radiation therapy. That being said, there are a number of questions that must be answered before we can appreciate the true clinical impact that LCT could have for the oligometastatic NSCLC population.

One such question is what the optimal dose and fractionation are for LCT. In the Gomez trial, the LCT group could receive SBRT, hypofractionated radiation therapy, or concurrent chemoradiation therapy as the dose and fractionation were at the discretion of the radiation oncologist, with the intent of delivering curative-intent treatment when possible (6). Additionally, some patients were treated with a combination of palliative-intent radiotherapy (to bone metastases) and definitive-intent 
Table 1 Current ongoing randomized controlled trials evaluating radiotherapy for local consolidation of all disease sites in oligometastatic NSCLC

\begin{tabular}{|c|c|c|c|c|c|}
\hline Study & Trial type/status & Number to accrue & Major inclusion criteria & Consolidative therapy approach & Primary endpoint \\
\hline SARON (UK) (8) & Phase III & 340 & $\begin{array}{l}\text { NSCLC with } 1-3 \\
\text { metastases (EGFR and } \\
\text { ALK negative) }\end{array}$ & Conventional RT or SBRT & Overall survival \\
\hline $\begin{array}{l}\text { STOP-NSCLC } \\
\text { (Canada) }\end{array}$ & Phase II & 54 & $\begin{array}{l}\text { NSCLC with 1-5 } \\
\text { metastases }\end{array}$ & SBRT & Overall survival \\
\hline HALT (UK) (9) & Phase II/III & 110 & $\begin{array}{l}\text { NSCLC with 1-3 } \\
\text { metastases showing } \\
\text { progression on TKI }\end{array}$ & SBRT and systemic TKI & $\begin{array}{l}\text { Progression free } \\
\text { survival }\end{array}$ \\
\hline $\begin{array}{l}\text { NORTHSTAR } \\
\text { (US) (10) }\end{array}$ & Phase II & 143 & $\begin{array}{l}\text { EGFR+ NSCLC, stage } \\
\text { IIIB/IV not amenable to } \\
\text { curative-intent therapy }\end{array}$ & $\begin{array}{l}\text { Osimertinib + surgery and/or RT } \\
\text { vs. osimertinib alone }\end{array}$ & $\begin{array}{l}\text { Progression free } \\
\text { survival }\end{array}$ \\
\hline
\end{tabular}

NSCLC, non-small cell lung cancer.

SBRT, or a combination of radiation therapy and surgery. By comparison, in SABR-COMET, only ablative doses of radiation could be used, and surgery was not allowed (5). With this in mind, understanding the associated toxicities will be key, as SABR-COMET showed a significantly higher rate of Grade 2 or greater adverse events, including treatment-related deaths in $5 \%$ of patients after SBRT.

These Phase II studies also included relatively small participant numbers (by design) that limit sub-group comparisons to determine which patients LCT benefits most, and heterogeneity becomes inevitable between treatment arms despite randomization. It is thus important that these findings be validated by large Phase III randomized controlled trials, such as those listed in Table 1. We believe that results from Phase III trials are necessary before LCT finds utility in standard clinical practice.

Additionally, enhanced tumor biomarkers, such as pre-and post-treatment circulating tumor DNA burden, may help us more accurately predict subgroup outcomes (11). Another important aspect will be the transparent reporting of workflows for these patients-especially those receiving SBRT to $\geq 3$ extracranial metastases. One can easily imagine the logistical issues of trying to simultaneously or sequentially treat lesions that are subject to motion (lung, liver) versus those that are not (bone, brain), in terms of setup and motion management. Careful design and analysis of clinical processes will be needed for physicians, physicists, dosimetrists and therapists to avoid errors (12). It is likely that new quality assurance pathways will need to be created for comprehensive oligometastatic SBRT cases. Finally, it remains to be determined whether there are any differences in patient-reported outcomes (PRO) and quality-of-life (QoL) assessments for patients treated with conventional therapy versus LCT. While we presume that increased time to progression of disease and resumption of systemic therapy would be associated with improved PRO/QoL outcomes, is there a threshold for the number of treatment sites where this does not hold true? Similarly, if we look at the history and legacy of phase III clinical trials for brain metastases, is it possible that there are survival benefits for some patient sub-populations but not others (13)? 
Prospective phase III trials such as those listed in Table 1 will help us find these answers, and international collaboration and support of these trials will be key to providing the best possible care to our patients with oligometastatic lung cancer.

\section{Acknowledgments}

We are grateful to patients and their families involved in the cited studies for making this research possible.

Funding: This work was supported by the National Cancer Institute of the U.S. National Institutes of Health under award number K08CA238711 (A. A. Chaudhuri) and the Cancer Research Foundation Young Investigator Award (A. A. Chaudhuri).

\section{Footnote}

Conflicts of Interest: CGR has research support from Varian Medical Systems, Elekta and Merck, consulting agreements with Varian Medical Systems, EMD Serono, AstraZeneca and Radialogica, and stocks with Radialogica. AAC reports speaker honoraria and travel support from Varian Medical Systems, Roche and Foundation Medicine, research support from Roche, and has served as an advisor/consultant for Tempus Labs, Roche, Geneoscopy, Fenix Group International and Oscar Health. The other authors have no conflicts of interest to declare.

Ethical Statement: The authors are accountable for all aspects of the work in ensuring that questions related to the accuracy or integrity of any part of the work are appropriately investigated and resolved.

\section{References}

1. Khan AJ, Mehta PS, Zusag TW, et al. Long term disease-free survival resulting from combined modality management of patients presenting with oligometastatic, non-small cell lung carcinoma (NSCLC). Radiother Oncol 2006;81:163-7.

2. Weichselbaum RR, Hellman S. Oligometastases revisited. Nat Rev Clin Oncol 2011;8:378-82.

3. Macdermed DM, Weichselbaum RR, Salama JK. A rationale for the targeted treatment of oligometastases with radiotherapy. J Surg Oncol 2008;98:202-6.

4. Gomez DR, Tang C, Zhang J, et al. Local Consolidative Therapy Vs. Maintenance Therapy or Observation for Patients With Oligometastatic Non-Small-Cell Lung
Cancer: Long-Term Results of a Multi-Institutional, Phase II, Randomized Study. J Clin Oncol 2019;37:1558-65.

5. Palma DA, Olson R, Harrow $S$, et al. Stereotactic ablative radiotherapy versus standard of care palliative treatment in patients with oligometastatic cancers (SABRCOMET): a randomised, phase 2, open-label trial. Lancet 2019;393:2051-8.

6. Gomez DR, Blumenschein GR Jr, Lee JJ, et al. Local consolidative therapy versus maintenance therapy or observation for patients with oligometastatic non-smallcell lung cancer without progression after first-line systemic therapy: a multicentre, randomised, controlled, phase 2 study. Lancet Oncol 2016;17:1672-82.

7. Palma DA, Olson R, Harrow S, et al. Stereotactic ablative radiotherapy for the comprehensive treatment of 4-10 oligometastatic tumors (SABR-COMET-10): study protocol for a randomized phase III trial. BMC Cancer 2019;19:816.

8. Conibear J, Chia B, Ngai Y, et al. Study protocol for the SARON trial: a multicentre, randomised controlled phase III trial comparing the addition of stereotactic ablative radiotherapy and radical radiotherapy with standard chemotherapy alone for oligometastatic non-small cell lung cancer. BMJ Open 2018;8:e20690.

9. McDonald F, Hanna GG. Oligoprogressive Oncogeneaddicted Lung Tumours: Does Stereotactic Body Radiotherapy Have a Role? Introducing the HALT Trial. Clin Oncol (R Coll Radiol) 2018;30:1-4.

10. Elamin YY, Antonoff M, Blakely C, et al. 1509TiP. Randomized phase II trial of osimertinib with or without local consolidation therapy (LCT) for patients with EGFR-mutant metastatic NSCLC (NORTHSTAR). Ann Oncol 2018;29:mdy292.130.

11. Chaudhuri AA, Chabon JJ, Lovejoy AF, et al. Early Detection of Molecular Residual Disease in Localized Lung Cancer by Circulating Tumor DNA Profiling. Cancer Discov 2017;7:1394-403.

12. Chera BS, Jackson M, Mazur LM, et al. Improving quality of patient care by improving daily practice in radiation oncology. Semin Radiat Oncol 2012;22:77-85.

13. Patchell RA, Tibbs PA, Walsh JW, et al. A randomized trial of surgery in the treatment of single metastases to the brain. N Engl J Med 1990;322:494-500.

Cite this article as: Samson PP, Spraker MB, Badiyan SN, Vlacich G, Robinson CG, Chaudhuri AA. Local consolidative therapy for oligometastatic non-small cell lung cancer. J Thorac Dis 2019;11(12):5649-5651. doi: 10.21037/jtd.2019.11.19 